\title{
BANDO-CALABI-FUTAKI CHARACTER OF COMPACT TORIC MANIFOLDS
}

\author{
Dedicated to Professor Tadao Oda on his sixtieth birthday \\ YASUHIRO NAKAGAWA
}

(Received November 24, 1999, revised August 28, 2000)

\begin{abstract}
The Bando-Calabi-Futaki character of a compact Kähler manifold is an obstruction to the existence of Kähler metrics with constant scalar curvature, which is a generalization of the Futaki character of a Fano manifold. In this paper, we study the BandoCalabi-Futaki character of a compact toric manifold. In particular, we shall prove that the Bando-Calabi-Futaki character of a compact toric manifold vanishes on the Lie algebra of the unipotent radical of the automorphism group.
\end{abstract}

1. Introduction. Let $X$ be a compact connected $r$-dimensional complex manifold and $\eta \in H^{2}(X ; \boldsymbol{R})$. We assume that $\eta$ is positive, that is, there exists a Kähler metric $g$ on $X$ such that its Kähler form

$$
\omega_{g}:=\sqrt{-1} \sum_{i, j=1}^{r} g_{i \bar{\jmath}} d z^{i} \wedge d \overline{z^{j}}
$$

represents $2 \pi \eta$ in the de Rham cohomology group $H_{D R}^{2}(X ; \boldsymbol{R})$, where $\left(z^{1}, z^{2}, \ldots, z^{r}\right)$ is a local holomorphic coordinate on $X$. We denote by $\operatorname{Aut}^{\circ}(X)$ the identity component of the group $\operatorname{Aut}(X)$ of holomorphic automorphisms of $X$, whose Lie algebra is identified with the Lie algebra $H^{0}\left(X ; \mathcal{O}\left(T^{1,0} X\right)\right)$ of holomorphic vector fields on $X$. Here $T^{1,0} X$ is the holomorphic vector bundle of tangent vectors of type $(1,0)$ on $X$. Recall that the Albanese map of $X$ to the Albanese variety $\operatorname{Alb}(X)$ naturally induces a Lie group homomorphism

$$
\alpha_{X}: \operatorname{Aut}^{\circ}(X) \rightarrow \operatorname{Aut}^{\circ}(\operatorname{Alb}(X)) \cong \operatorname{Alb}(X) .
$$

Let $G_{X}$ be the identity component of the kernel of the homomorphism $\alpha_{X}$, and $\mathfrak{g}_{X}$ the corresponding Lie subalgebra of $H^{0}\left(X ; \mathcal{O}\left(T^{1,0} X\right)\right)$. Then, by a theorem of Fujiki [6], $G_{X}$ has a natural structure of a linear algebraic group (defined over $\boldsymbol{C}$ ). We denote by $U_{X}$ the unipotent radical of $G_{X}$. More generally, we consider a linear algebraic group $G$ (defined over $\boldsymbol{C}$ ) and a homomorphism $\rho: G \rightarrow \operatorname{Aut}(X)$ of algebraic groups. By $\rho_{*}: \mathfrak{g} \rightarrow H^{0}\left(X ; \mathcal{O}\left(T^{1,0} X\right)\right)$, we denote the Lie algebra homomorphism induced from $\rho$, where $\mathfrak{g}:=\operatorname{Lie}(G)$ is the Lie algebra of $G$.

2000 Mathematics Subject Classification. Primary 32Q20; Secondary 14M25, 32M05.

Partly supported by the Grant-in-Aid for Encouragement of Young Scientists (No. 09740047), The Ministry of Education, Science, Sports and Culture, Japan. 
REMARK 1.1. (i) If $X$ is Fano, i.e., the first Chern class $c_{1}(X)$ of $X$ is positive, then $G_{X}=\operatorname{Aut}^{\circ}(X)$.

(ii) If $X$ is an $r$-dimensional compact toric manifold, that is, $X$ is an $r$-dimensional compact irreducible non-singular variety defined over $C$ with an almost-homogeneous algebraic action of an $r$-dimensional algebraic torus $T_{r}:=\left(C^{*}\right)^{r}$, then $G_{X}=\operatorname{Aut}^{\circ}(X)$.

By $\operatorname{Ric}_{g}$ and $s_{g}$, we denote the Ricci form and the scalar curvature of $g$, respectively, namely, we put

$$
\begin{aligned}
& \operatorname{Ric}_{g}=\sqrt{-1} \sum_{i, j=1}^{r} R_{i \bar{\jmath}} d z^{i} \wedge d \overline{z^{j}}:=-\sqrt{-1} \partial \bar{\partial} \log \operatorname{det}\left(g_{i \bar{\jmath}}\right), \\
& s_{g}:=\sum_{i, j=1}^{r} g^{\bar{j} i} R_{i \bar{\jmath}}
\end{aligned}
$$

where $\left(g^{\bar{j}}\right)$ is the inverse matrix of $\left(g_{i \bar{J}}\right)$. By means of the harmonic integration theory, there exists a real-valued $C^{\infty}$ function $f \in C^{\infty}(X)_{R}$ such that

$$
s_{g}-r \mu_{\eta}=\square_{g} f_{g},
$$

where $\square_{g}:=\sum_{i, j=1}^{r} g^{\bar{j}}\left(\partial^{2} / \partial z^{i} \partial \overline{z^{j}}\right)$ is the complex Laplacian for functions on the Kähler manifold $(X, g)$, and $\mu_{\eta} \in \boldsymbol{R}$ is the constant defined by

$$
\mu_{\eta}:=\frac{\left(c_{1}(X) \cup \eta^{r-1}\right)[X]}{\eta^{r}[X]}=\frac{\int_{X} s_{g}\left(\frac{\omega_{g}}{2 \pi}\right)^{r}}{r \int_{X}\left(\frac{\omega_{g}}{2 \pi}\right)^{r}} \in \boldsymbol{R} .
$$

Bando [2 ], Calabi [ 4 ] and Futaki [9] defined an obstruction to the existence of Kähler metrics with constant scalar curvature as follows:

Definition 1.3 (Bando [2], Calabi [4] and Futaki [9]). A linear functional $F_{X}^{\eta}: H^{0}\left(X ; \mathcal{O}\left(T^{1,0} X\right)\right) \rightarrow C$ defined by

$$
F_{X}^{\eta}(V):=\frac{1}{\sqrt{-1}} \int_{X}\left(V f_{g}\right)\left(\frac{\omega_{g}}{2 \pi}\right)^{r}, \quad V \in H^{0}\left(X ; \mathcal{O}\left(T^{1,0} X\right)\right),
$$

is called the Bando-Calabi-Futaki character of $(X, \eta)$.

We now recall the following fundamental facts about the Bando-Calabi-Futaki characters:

FACT 1.4 (Bando [2], Calabi [4] and Futaki [9]). Let $X$ and $\eta$ be as above. Then we have the following:

(i) $F_{X}^{\eta}$ does not depend on the choice of $g$ satisfying $\left[\omega_{g}\right]=2 \pi \eta$.

(ii) If $X$ admits a Kähler metric $g$ with constant scalar curvature satisfying $\left[\omega_{g}\right]=2 \pi \eta$, then $F_{X}^{\eta}$ vanishes. 
(iii) $F_{X}^{\eta}$ is a Lie algebra character of $H^{0}\left(X ; \mathcal{O}\left(T^{1,0} X\right)\right)$, that is,

$$
\left.F_{X}^{\eta}\right|_{\left[H^{0}\left(X ; \mathcal{O}\left(T^{1,0} X\right)\right), H^{0}\left(X ; \mathcal{O}\left(T^{1,0} X\right)\right)\right]} \equiv 0 .
$$

REMARK 1.5. If $\eta$ is the first Chern class $c_{1}(X)$ of $X$, then the Bando-Calabi-Futaki character $F_{X}^{c_{1}(X)}$ coincides with the original Futaki character, which was introduced in [8] as an obstruction to the existence of Einstein-Kähler metrics.

DEFinition 1.6. Let $\pi_{E}: E \rightarrow X$ be a holomorphic vector bundle of rank $k$ over $X$. We say that $E$ is $(G, \rho)$-linearized if $G$ acts on $E$ biregularly in such a way that

(i) $\pi_{E} \circ \gamma=\rho(\gamma) \circ \pi_{E}$ for any $\gamma \in G$;

(ii) for any $\gamma \in G$ and $p \in X$,

$$
\left.\gamma\right|_{E_{p}}: E_{p} \rightarrow E_{\rho(\gamma)(p)}
$$

is a $C$-linear map, where $E_{p}:=\pi_{E}^{-1}(p)$ is the fiber of $\pi_{E}$ at $p \in X$.

Furthermore, if $G$ is a subgroup of $\operatorname{Aut}(X)$ and $\rho$ is the inclusion map, then we simply say that $E$ is $G$-linearized.

In [15], the author proved the following:

FACT 1.7 (Nakagawa [15]). Let $X$ and $\eta$ be as above. We assume that there exists a holomorphic line bundle $L$ over $X$ such that $L$ is $G_{X}$-linearized and $c_{1}(L)=\eta$, where $c_{1}(L)$ is the first Chern class of L. Then

$$
\left.F_{X}^{\eta}\right|_{\mathfrak{u}_{X}} \equiv 0,
$$

where $\mathfrak{u}_{X}:=\operatorname{Lie}\left(U_{X}\right)$ is the Lie algebra of $U_{X}$.

The main purpose of this paper is to generalize this fact to the case of a more general situation, that is, we shall prove the following theorem:

THEOREM 1.8. Let $X, \eta, G$ and $\rho$ be as above. We assume that there exists a holomorphic line bundle $L$ over $X$ such that $L$ is $(G, \rho)$-linearized and $c_{1}(L)=\eta$. Then

$$
\left.\left(F_{X}^{\eta} \circ \rho_{*}\right)\right|_{\mathfrak{u}} \equiv 0
$$

for any unipotent subgroup $U \subseteq G$ with Lie algebra $\mathfrak{u}:=\operatorname{Lie}(U)$.

As an application of this theorem, we shall also prove the following theorem:

THEOREM 1.9. Let $X$ be an $r$-dimensional compact toric manifold. By definition, an $r$-dimensional algebraic torus $T_{r}:=\left(C^{*}\right)^{r}$ acts on $X$ biholomorphically; hence the Lie algebra $\mathfrak{t}_{r}:=\operatorname{Lie}\left(T_{r}\right)$ of $T_{r}$ is regarded as a Lie subalgebra of $H^{0}\left(X ; \mathcal{O}\left(T^{1,0} X\right)\right)$. If $\eta \in$ $H^{2}(X ; Z)$ is positive, then the following are equivalent, without any assumptions concerning a linearization of the natural action of $\operatorname{Aut}(X)$ on $X$ :

(i) $F_{X}^{\eta}$ vanishes identically on $H^{0}\left(X ; \mathcal{O}\left(T^{1,0} X\right)\right)$.

(ii) $F_{X}^{\eta}$ vanishes on $\mathfrak{t}_{r}$.

Acknowledgments. Part of this paper was written when the author participated in the "Programme on Lie Groups, March-June 1999” at the Institute of Mathematical Research, the University of Hong Kong. He wishes to thank Professor Ngaiming Mok who invited him to 
the University of Hong Kong. He also wishes to thank the Department of Mathematics, the University of Hong Kong, for constant assistance all through his stay in Hong Kong.

\section{Bando-Calabi-Futaki characters as holomorphic invariants (Proof of Theorem}

1.8). Throughout this section, we fix a compact connected $r$-dimensional complex manifold $X$, a positive class $\eta \in H^{2}(X ; \boldsymbol{R})$, a linear algebraic group $G$ (defined over $\boldsymbol{C}$ ) and a homomorphism $\rho: G \rightarrow \operatorname{Aut}(X)$ of algebraic groups. Let $\pi_{E}: E \rightarrow X$ be a holomorphic vector bundle of rank $k$ over $X$. We assume that $E$ is $(G, \rho)$-linearized. Then, for any $V \in \mathfrak{g}$, a holomorphic action (see [3])

$$
\Lambda_{V}^{E}: A^{0}(E) \rightarrow A^{0}(E),
$$

of $V$ on $E$ is induced, that is, $\Lambda_{V}^{E}$ satisfies the following properties:

(i) $\Lambda_{V}^{E}$ is a $\boldsymbol{C}$-linear map.

(ii) For all $\psi \in C^{\infty}(X)_{C}$ and $s \in A^{0}(E)$,

$$
\Lambda_{V}^{E}(\psi s)=\left(\left(\rho_{*} V\right) \psi\right) s+\psi \Lambda_{V}^{E} s .
$$

(iii) $\Lambda_{V}^{E}$ commutes with $\bar{\partial}$, i.e., $\bar{\partial} \Lambda_{V}^{E}=\Lambda_{V}^{E} \bar{\partial}$.

Here we denote by $A^{p}(E)$ the space of $E$-valued $p$-forms on $X$ for $p=0,1, \ldots, r$.

EXAmple 2.1. $E=T^{1,0} X$ is canonically $\operatorname{Aut}(X)$-linearized. In this case, $\Lambda_{V}^{T^{1,0} X}$ is the Lie differentiation $L_{V}$ of vector fields with respect to a holomorphic vector field $V \in$ $H^{0}\left(X ; \mathcal{O}\left(T^{1,0} X\right)\right)$ on $X$.

Let $h$ be a Hermitian metric on $E$ and $\nabla^{h}: A^{0}(E) \rightarrow A^{1}(E)$ the Hermitian connection of $h$ (see for instance [12, p. 12]). We define the curvature $\Theta_{h}$ of $\nabla^{h}$ by

$$
\Theta_{h}:=\bar{\partial}\left(h^{-1} \partial h\right) \in A^{2}(\operatorname{End}(E)),
$$

where $\operatorname{End}(E)$ is the endomorphism bundle of $E$ over $X$. For each $V \in \mathfrak{g}$, we put $\mathcal{L}_{V}^{(E, h)}:=$ $\nabla_{\rho_{*} V}^{h}-\Lambda_{V}^{E} \in A^{0}(\operatorname{End}(E))$. Let $l \in \boldsymbol{Z}_{\geq 0}$ be a non-negative integer and $\phi$ a $G L(k, \boldsymbol{C})$ invariant symmetric polynomial of degree $r+l$ on $\mathfrak{g l}(k, \boldsymbol{C})$ (see [10, p. 21]). For example, $c_{1}^{r+l}:=((\sqrt{-1} / 2 \pi) \operatorname{tr})^{r+l}$ is a $G L(k, C)$-invariant symmetric polynomial of degree $r+l$ on $\mathfrak{g l}(k, \boldsymbol{C})$. We now define a map $\mathcal{C}_{E}^{\phi}: \mathfrak{g} \rightarrow \boldsymbol{C}$ by

$$
\mathcal{C}_{E}^{\phi}(V):=\int_{X} \phi\left(\mathcal{L}_{V}^{(E, h)}+\Theta_{h}\right), \quad V \in \mathfrak{g} .
$$

For this map $\mathcal{C}_{E}^{\phi}$, we can prove the following facts:

FACT 2.2 (cf. Futaki and Morita [11]). Let $X,(E, h)$ and $\phi$ be as above. Then we have the following:

(i) $\mathcal{C}_{E}^{\phi}$ dose not depend on the choice of a Hermitian metric $h$ on $E$, i.e., $\mathcal{C}_{E}^{\phi}$ is a holomorphic invariant of $(X, E)$.

(ii) $\mathcal{C}_{E}^{\phi}$ is a $G$-invariant symmetric polynomial of degree l on $\mathfrak{g}$. In particular, if $l=1$, then $\mathcal{C}_{E}^{\phi}$ is a character of the Lie algebra $\mathfrak{g}$. 
(iii) For any $V \in H^{0}\left(X ; \mathcal{O}\left(T^{1,0} X\right)\right)$,

$$
F_{X}^{c_{1}(X)}(V)=-\frac{2 \pi}{r+1} \mathcal{C}_{T^{1,0} X}^{c^{r+1}}(V)=-\frac{2 \pi}{r+1} \mathcal{C}_{K_{X}^{-1}}^{c_{1}^{r+1}}(V),
$$

where $T^{1,0} X$ and $K_{X}^{-1}:=\operatorname{det} T^{1,0} X=\wedge^{r} T^{1,0} X$ are regarded as $\operatorname{Aut}(X)$-linearized bundles over $X$ in terms of the canonical $\operatorname{Aut}(X)$-actions on them.

Let $g^{\prime}$ be an arbitrary Hermitian metric on $X$. For $V \in \mathfrak{g}$, if a point $p \in X$ is a zero point of $\rho_{*} V \in H^{0}\left(X ; \mathcal{O}\left(T^{1,0} X\right)\right)$, then $\mathcal{L}_{\rho * V}^{\left(T^{1,0} X, g^{\prime}\right)}$ induces the linear map

$$
\mathcal{L}_{\rho_{*} V, p}^{\left(T^{1,0} X, g^{\prime}\right)}=-\left(L_{\rho_{*} V}\right)_{p}: T_{p}^{1,0} X \rightarrow T_{p}^{1,0} X
$$

$V \in \mathfrak{g}$ is said to be non-degenerate if the following two conditions hold:

(i) The zero set $\operatorname{Zero}\left(\rho_{*} V\right)$ of $\rho_{*} V$ is finite.

(ii) For each zero point $p \in \operatorname{Zero}\left(\rho_{*} V\right)$ of $\rho_{*} V$, the linear map

$$
\mathcal{L}_{\rho_{*} V, p}^{\left(T^{1,0} X, g^{\prime}\right)}: T_{p}^{1,0} X \rightarrow T_{p}^{1,0} X
$$

is non-singular.

The following localization formula for $\mathcal{C}_{E}^{\phi}$ allows us to calculate explicitly the BandoCalabi-Futaki character of a compact toric manifold (see Corollary 4.6):

FACT 2.3 (Bott [3]). Let $X,(E, h)$ and $\phi$ be as above, and $V \in \mathfrak{g}$ a non-degenerate element. Then we have

$$
\mathcal{C}_{E}^{\phi}(V)=\sum_{p \in \operatorname{Zero}(V)} \frac{\phi\left(\mathcal{L}_{V, p}^{(E, h)}\right)}{\operatorname{det} \frac{\sqrt{-1}}{2 \pi} \mathcal{L}_{\rho_{*} V, p}^{\left(T^{1,0} X, g^{\prime}\right)}},
$$

where $g^{\prime}$ is an arbitrary Hermitian metric on $X$.

Now, we assume that there exists a holomorphic line bundle $L$ over $X$ such that $L$ is $(G, \rho)$-linearized and $c_{1}(L)=\eta$. Under this assumption, an argument similar to that in [17, Section 6] allows us to prove the following Tian's formula for the Bando-Calabi-Futaki character (see also [15, Section 3]):

THEOREM 2.4 (Tian [17]). Let $X, \eta, G, \rho$ and $L$ be as above. Then, for any integer $\delta \in \boldsymbol{Z}$ and $V \in \mathfrak{g}$, we have

$$
\begin{aligned}
F_{X}^{\eta}\left(\rho_{*} V\right)= & -\frac{2 \pi}{2^{r}(r+1) !} \sum_{j=0}^{r}(-1)^{j}\left(\begin{array}{l}
r \\
j
\end{array}\right) \mathcal{C}_{K_{X}^{-1} \otimes L^{\delta+r-2 j}}^{c_{1}^{r+1}}(V) \\
& +2 \pi\left(\delta+\frac{r \mu_{\eta}}{r+1}\right) \mathcal{C}_{L}^{c_{1}^{r+1}}(V),
\end{aligned}
$$

where $L^{\delta+r-2 j}:=L^{\otimes(\delta+r-2 j)}$ is the $(\delta+r-2 j)$-th tensor power of $L$. Here we regard $K_{X}^{-1} \otimes L^{\delta+r-2 j}, j=0,1, \ldots, r$, as $(G, \rho)$-linearized line bundles by the canonical Aut $(X)$ action on $K_{X}^{-1}$. 
Together with this Tian's formula, the following fact implies Theorem 1.8 by the same argument as that in [15, Section 4]:

FACT 2.5 (Mabuchi [13]). Let $X, G, \rho$ and $L$ be as above. Then, for any unipotent subgroup $U$ of $G, \mathcal{C}_{L}^{c_{1}^{n+1}}$ vanishes on the Lie algebra $\mathfrak{u}:=\operatorname{Lie}(U)$ of $U$.

3. Bando-Calabi-Futaki character of compact toric manifolds (Proof of Theorem 1.9). First, we recall some basic notions and facts concerning toric manifolds (see [16] for more details). Let $T_{r}:=\left(\boldsymbol{C}^{*}\right)^{r}$ be an $r$-dimensional algebraic torus. We put $N:=\boldsymbol{Z}^{r}$ and $M:=\operatorname{Hom}_{\boldsymbol{Z}}(N, \boldsymbol{Z})\left(\cong \boldsymbol{Z}^{r}\right)$, where we regard elements of $N$ and $M$ as $r$-dimensional column vectors and row vectors, respectively. Let $\Sigma$ be a complete non-singular fan in $N$ (see [16] for the definition of a complete non-singular fan) and $\Sigma(i)$ the set of $i$-dimensional cones in $\Sigma$ for $i=0,1, \ldots, r$. We denote by $X_{\Sigma}$ the $r$-dimensional compact toric manifold associated with $\Sigma$. Then $T_{r}$ acts on $X_{\Sigma}$ biholomorphically, and $X_{\Sigma}$ has an open dense $T_{r}$-orbit $\mathfrak{O}_{\Sigma}$ isomorphic to $T_{r}$.

FACT 3.1 (Cox [5]). Let $\Sigma$ be a complete non-singular fan in $N$ and $d_{\Sigma}:=\# \Sigma(1)$ the number of the one-dimensional cones in $\Sigma$. Then:

(i) There exists a $\left(d_{\Sigma}-r\right)$-dimensional algebraic subtorus $H_{\Sigma}$ of $\left(\boldsymbol{C}^{*}\right)^{d_{\Sigma}}$ and an $H_{\Sigma}$-invariant open subset $\mathcal{W}_{\Sigma}$ of $\boldsymbol{C}^{d_{\Sigma}}$ such that $H_{\Sigma}$ acts freely on $\mathcal{W}_{\Sigma}$ and

$$
X_{\Sigma}=\mathcal{W}_{\Sigma} / H_{\Sigma}
$$

Here the $H_{\Sigma}$-action on $\boldsymbol{C}^{d_{\Sigma}}$ is induced from the canonical $\left(\boldsymbol{C}^{*}\right)^{d_{\Sigma}}$-action on $\boldsymbol{C}^{d_{\Sigma}}$.

(ii) Let $\widetilde{G}_{\Sigma}$ be the centralizer of $H_{\Sigma}$ in $\operatorname{Aut}\left(\mathcal{W}_{\Sigma}\right)$. Then

$$
\operatorname{Aut}^{\circ}\left(X_{\Sigma}\right) \cong \widetilde{G}_{\Sigma} / H_{\Sigma} \text {. }
$$

(iii) $\widetilde{G}_{\Sigma}$ and $\operatorname{Aut}^{\circ}\left(X_{\Sigma}\right)$ are connected linear algebraic groups (defined over $\boldsymbol{C}$ ). Let $\widetilde{U}_{\Sigma}$ and $U_{\Sigma}$ be the unipotent radicals of $\widetilde{G}_{\Sigma}$ and $\operatorname{Aut}^{\circ}\left(X_{\Sigma}\right)$, respectively. Then

$$
\left.\rho_{\Sigma}\right|_{\widetilde{U}_{\Sigma}}: \widetilde{U}_{\Sigma} \rightarrow U_{\Sigma}
$$

is an isomorphism, where $\rho_{\Sigma}: \widetilde{G}_{\Sigma} \rightarrow \operatorname{Aut}^{\circ}\left(X_{\Sigma}\right)$ is the natural projection induced by the isomorphism $\operatorname{Aut}^{\circ}\left(X_{\Sigma}\right) \cong \widetilde{G}_{\Sigma} / H_{\Sigma}$. Furthermore, there exists a reductive algebraic subgroup $R_{\Sigma}$ of $\operatorname{Aut}^{\circ}\left(X_{\Sigma}\right)$ with $T_{r}$ as a maximal algebraic torus such that

$$
\operatorname{Aut}^{\circ}\left(X_{\Sigma}\right)=R_{\Sigma} \ltimes U_{\Sigma} .
$$

EXAMPLE 3.2. A typical example of an $r$-dimensional compact toric manifold is the $r$-dimensional complex projective space $\boldsymbol{P}^{r}(\boldsymbol{C})$. If $X_{\Sigma}=\boldsymbol{P}^{r}(\boldsymbol{C})$, then we have:

$$
\begin{aligned}
& d_{\Sigma}=r+1, \\
& H_{\Sigma}=\left\{(t, t, \ldots, t) \in\left(\boldsymbol{C}^{*}\right)^{r+1} ; t \in \boldsymbol{C}^{*}\right\} \cong \boldsymbol{C}^{*}, \\
& \mathcal{W}_{\Sigma}=\boldsymbol{C}^{r+1} \backslash\{0\}, \\
& \widetilde{G}_{\Sigma}=G L(r+1, \boldsymbol{C}), \\
& \operatorname{Aut}^{\circ}\left(X_{\Sigma}\right)=\operatorname{Aut}\left(X_{\Sigma}\right)=P G L(r+1, \boldsymbol{C}) .
\end{aligned}
$$


To each $v \in \Sigma(1)$, there corresponds a $T_{r}$-invariant Weil divisor $D_{v}$ on $X_{\Sigma}$. More generally, a map $\alpha: \Sigma(1) \rightarrow \boldsymbol{Z}$ defines a $T_{r}$-invariant Weil divisor $D(\alpha):=-\sum_{v \in \Sigma(1)} \alpha(v) D_{v}$, and we denote by $L_{\alpha}$ the $T_{r}$-linearized holomorphic line bundle over $X_{\Sigma}$ corresponding to $D(\alpha)$, i.e., $L_{\alpha}=\mathcal{O}(D(\alpha))$.

EXAMPLE 3.3. Let $\Sigma$ be a complete non-singular fan in $N$ and $X_{\Sigma}$ the compact toric manifold associated with $\Sigma$. Then the anti-canonical line bundle $K_{X_{\Sigma}}^{-1}$ of $X_{\Sigma}$ corresponds to the map

$$
\alpha_{0}: \Sigma(1) \ni v \mapsto-1 \in \boldsymbol{Z},
$$

that is, $K_{X_{\Sigma}}^{-1}$ corresponds to the $T_{r}$-invariant Weil divisor $\sum_{v \in \Sigma(1)} D_{\nu}$.

If $L_{\alpha}$ is ample, that is, $c_{1}\left(L_{\alpha}\right) \in H^{2}\left(X_{\Sigma} ; Z\right)$ is positive, then we say that $\alpha$ is ample. Let $\Sigma(1)=\left\{v_{1}, v_{2}, \ldots, v_{d_{\Sigma}}\right\}$ and put $\alpha_{i}:=\alpha\left(v_{i}\right) \in \boldsymbol{Z}$ for $i=1,2, \ldots, d_{\Sigma}$. Then we define a character $\lambda_{\alpha}:\left(\boldsymbol{C}^{*}\right)^{d_{\Sigma}} \rightarrow \boldsymbol{C}^{*}$ of $\left(\boldsymbol{C}^{*}\right)^{d_{\Sigma}}$ by $\lambda_{\alpha}\left(s_{1}, s_{2}, \ldots, s_{d_{\Sigma}}\right):=s_{1}^{\alpha_{1}} s_{2}^{\alpha_{2}} \ldots s_{d_{\Sigma}}^{\alpha_{d_{\Sigma}}} \cdot H_{\Sigma}$ acts on $\mathcal{W}_{\Sigma} \times \boldsymbol{C}$ by

$$
k:(z, \xi) \mapsto\left(k \cdot z, \lambda_{\alpha}(k)^{-1} \xi\right),
$$

where $k \in H_{\Sigma}, z \in \mathcal{W}_{\Sigma}$ and $\xi \in \boldsymbol{C}$.

FACT 3.4 (cf. Audin [1, Chapter VI]). The projection $\mathcal{W}_{\Sigma} \longrightarrow X_{\Sigma}$ is a principal $H_{\Sigma}$-bundle. Furthermore, the $T_{r}$-linearized holomorphic line bundle $L_{\alpha}$ over $X_{\Sigma}$ is given by

$$
L_{\alpha}=\mathcal{W}_{\Sigma} \times \lambda_{\alpha} \boldsymbol{C}:=\left(\mathcal{W}_{\Sigma} \times \boldsymbol{C}\right) / H_{\Sigma}
$$

Proposition 3.5. For $\alpha$ as above, $L_{\alpha}$ is the $\left(\widetilde{G}_{\Sigma}, \rho_{\Sigma}\right)$-linearized holomorphic line bundle over $X_{\Sigma}$.

PROOF. The natural $\widetilde{G}_{\Sigma}$-action on $\mathcal{W}_{\Sigma}$ commutes with the $H_{\Sigma}$-action on $\mathcal{W}_{\Sigma}$. Then, by means of Fact 3.4, $\widetilde{G}_{\Sigma}$ acts on $L_{\alpha}=\mathcal{W}_{\Sigma} \times_{\lambda_{\alpha}} \boldsymbol{C}$ and $L_{\alpha}$ is $\left(\widetilde{G}_{\Sigma}, \rho_{\Sigma}\right)$-linearized.

For any $\eta \in H^{2}\left(X_{\Sigma} ; \boldsymbol{Z}\right)$, in view of [ 7, Section 3.4], there exists a map $\alpha_{\eta}: \Sigma(1) \rightarrow \boldsymbol{Z}$ such that $c_{1}\left(L_{\alpha_{\eta}}\right)=\eta$. Therefore, Theorem 1.8 together with Fact 3.1 (iii) and Proposition 3.5 implies the following theorem:

THEOREM 3.6. Let $\Sigma$ be a complete non-singular fan in $N$ and $\eta \in H^{2}\left(X_{\Sigma} ; Z\right) a$ positive class. Then the Bando-Calabi-Futaki character $F_{X_{\Sigma}}^{\eta}$ of $\left(X_{\Sigma}, \eta\right)$ vanishes on the Lie algebra $\mathfrak{u}_{\Sigma}:=\operatorname{Lie}\left(U_{\Sigma}\right)$ of $U_{\Sigma}$.

Recall that, for a reductive algebraic group $R$,

$$
\operatorname{Lie}(R)=\operatorname{Lie}(\operatorname{Center}(R))+[\operatorname{Lie}(R), \operatorname{Lie}(R)],
$$

and $\operatorname{Lie}(\operatorname{Center}(R)) \subseteq \operatorname{Lie}(T)$ for every maximal algebraic torus $T$ of $R$, where $\operatorname{Center}(R)$ is the center of $R$. Since $R_{\Sigma}$ is reductive, Theorem 3.6 together with Fact 3.1 (iii) and (3.7) immediately implies Theorem 1.9. 
4. A combinatorial formula for the Bando-Calabi-Futaki character of compact toric manifolds. In [14], the author established a combinatorial formula for the Futaki character of a toric Fano manifold. In this section, we shall also establish a combinatorial formula for the Bando-Calabi-Futaki character of a compact toric manifold by the same argument as in [14].

Throughout this section, we fix a complete non-singular fan $\Sigma$ in $N:=Z^{r}$ and a positive class $\eta \in H^{2}\left(X_{\Sigma} ; \boldsymbol{Z}\right)$. We shall use the same notation as that in Section 3.

We define a basis $\left\{\tau_{i}:=t^{i}\left(\partial / \partial t^{i}\right) ; i=1,2, \ldots, r\right\}$ of the Lie algebra $\mathfrak{t}_{r}$ of $T_{r}$, where $\left(t^{1}, t^{2}, \ldots, t^{r}\right)$ is the standard coordinate for $T_{r}=\left(C^{*}\right)^{r}$. Note that we can regard $\mathfrak{t}_{r}$ as a complex Lie subalgebra of $H^{0}\left(X_{\Sigma} ; \mathcal{O}\left(T^{1,0} X_{\Sigma}\right)\right)$. For each $\sigma \in \Sigma(r)$ and $S \in G L(r, C)$, let

$$
a_{1}(\sigma)=\left(\begin{array}{c}
a_{1}^{1}(\sigma) \\
a_{1}^{2}(\sigma) \\
\vdots \\
a_{1}^{r}(\sigma)
\end{array}\right), \ldots, a_{r}(\sigma)=\left(\begin{array}{c}
a_{r}^{1}(\sigma) \\
a_{r}^{2}(\sigma) \\
\vdots \\
a_{r}^{r}(\sigma)
\end{array}\right) \in N
$$

be the generator of $\sigma$. We put

$$
\begin{aligned}
& A(\sigma):=\left(a_{1}(\sigma), \quad a_{2}(\sigma), \quad \ldots, \quad a_{r}(\sigma)\right) \\
& =\left(\begin{array}{cccc}
a_{1}^{1}(\sigma) & a_{2}^{1}(\sigma) & \ldots & a_{r}^{1}(\sigma) \\
a_{1}^{2}(\sigma) & a_{2}^{2}(\sigma) & \ldots & a_{r}^{2}(\sigma) \\
\vdots & \vdots & \ldots & \vdots \\
a_{1}^{r}(\sigma) & a_{2}^{r}(\sigma) & \ldots & a_{r}^{r}(\sigma)
\end{array}\right) \in G L(r, \boldsymbol{Z})
\end{aligned}
$$

and $Q(S ; \sigma)=\left(q_{j}^{i}(S ; \sigma)\right):=A(\sigma)^{-1} S \in G L(r, \boldsymbol{C})$. A non-singular matrix $S \in G L(r, \boldsymbol{C})$ is said to be non-degenerate if $S$ satisfies $q_{j}^{i}(S ; \sigma) \neq 0$ for all $i, j=1,2, \ldots, r$, and $\sigma \in \Sigma(r)$.

EXAMPLE 4.1. For example, a non-singular matrix

$$
S_{0}:=\left(\begin{array}{cccc}
1 & 1 & \ldots & 1 \\
\pi & \pi^{2} & \ldots & \pi^{r} \\
\pi^{2} & \pi^{4} & \ldots & \pi^{2 r} \\
\vdots & \vdots & \ldots & \vdots \\
\pi^{r-1} & \pi^{2(r-1)} & \ldots & \pi^{r(r-1)}
\end{array}\right) \in G L(r, C)
$$

is non-degenerate.

For $S=\left(s_{i}^{j}\right) \in G L(r, C)$ and $i=1,2, \ldots, r$, we define a holomorphic vector field $V_{i}(S):=\sum_{i=1}^{r} s_{i}^{j} \tau_{j}$ on $X_{\Sigma}$. Then $\left\{V_{i}(S) ; i=1,2, \ldots, r\right\}$ is a basis of $\mathfrak{t}_{r}$. For a map $\alpha: \Sigma(1) \rightarrow Z$, we define constants $\beta_{i}(S ; \sigma, \alpha), i=1,2, \ldots, r$, by

$$
\beta_{i}(S ; \sigma, \alpha):=\sum_{j=1}^{r} \alpha\left(\left\langle a_{j}(\sigma)\right\rangle\right) q_{i}^{j}(S ; \sigma),
$$

where $\left\langle a_{i}(\sigma)\right\rangle \in \Sigma(1)$ is the one-dimensional cone generated by $a_{i}(\sigma) \in N$. We put $b_{i}(\sigma, \alpha):=\beta\left(I_{r} ; \sigma, \alpha\right)$, where $I_{r} \in G L(r, \boldsymbol{C})$ is the identity matrix. 
In terms of the notation as above, we can establish the following combinatorial formula for $\mathcal{C}_{L_{\alpha}}^{c_{1}^{r+l}}\left(V_{i}(S)\right)$ :

THEOREM 4.2. Let $X_{\Sigma}$ be an $r$-dimensional compact toric manifold associated with a complete non-singular fan $\Sigma$ and $S \in G L(r, \boldsymbol{C})$ a non-degenerate non-singular matrix. Then we have

$$
\mathcal{C}_{L_{\alpha}}^{c_{1}^{r+l}}\left(V_{i}(S)\right)=\left(\frac{\sqrt{-1}}{2 \pi}\right)^{l} \sum_{\sigma \in \Sigma(r)} \frac{\beta_{i}(S ; \sigma, \alpha)^{r+l}}{\prod_{j=1}^{r} q_{i}^{j}(S ; \sigma)}
$$

for any $\alpha: \Sigma(1) \rightarrow Z, l \in Z_{\geq 0}$ and $i=1,2, \ldots, r$, where we regard $L_{\alpha}$ as a $T_{r}$-linearized holomorphic line bundle over $X_{\Sigma}$.

Proof. For each $\sigma \in \Sigma(r)$, there exists a $T_{r}$-invariant open subset $W_{\sigma}$ of $X_{\Sigma}$ such that $W_{\sigma} \cong \boldsymbol{C}^{r}$ and $X_{\Sigma}=\bigcup_{\sigma \in \Sigma(r)} W_{\sigma}$. Let $\left(t^{1}, t^{2}, \ldots, t^{r}\right)$ and $\left(z^{1}(\sigma), z^{2}(\sigma), \ldots, z^{r}(\sigma)\right)$ be the coordinate systems on $\mathfrak{O}_{\Sigma} \cong T_{r}=\left(\boldsymbol{C}^{*}\right)^{r}$ and $W_{\sigma} \cong \boldsymbol{C}^{r}$, respectively. The following system of identities is the coordinate transformation between these coordinates:

$$
t^{i}=\prod_{j=1}^{r} z^{j}(\sigma)^{a_{j}^{i}(\sigma)}, \quad i=1,2, \ldots, r .
$$

From these identities, for every $\sigma \in \Sigma(r)$ and $i=1,2, \ldots, r$, we have

$$
V_{i}(S)=\sum_{k=1}^{r} q_{i}^{k}(S ; \sigma) z^{k}(\sigma) \frac{\partial}{\partial z^{k}(\sigma)}
$$

on $W_{\sigma}$. In view of this expression of $V_{i}(S)$ and the non-degeneracy of $S$, we obtain

$$
\operatorname{Zero}\left(V_{i}(S)\right)=\left\{\text { the origin } o(\sigma) \text { of } W_{\sigma} \cong \boldsymbol{C}^{r} ; \sigma \in \Sigma(r)\right\}
$$

for $i=1,2, \ldots, r$. For each $\sigma \in \Sigma(r)$, the $T_{r}$-linearized holomorphic line bundle $L_{\alpha}$ over $X_{\Sigma}$ is trivialized on $W_{\sigma}$. In terms of this trivialization, the $T_{r}$-action on $\left.L_{\alpha}\right|_{W_{\sigma}}=W_{\sigma} \times \boldsymbol{C}$ is given by

$$
t: W_{\sigma} \times \boldsymbol{C} \ni(z, \xi) \mapsto\left(t \cdot z, \prod_{i=1}^{r}\left(t^{i}\right)^{-b_{i}(\sigma, \alpha)} \xi\right) \in W_{\sigma} \times \boldsymbol{C},
$$

where $t=\left(t^{1}, t^{2}, \ldots, t^{r}\right) \in T_{r}$ (see [16, p. 69]). Hence, for $\sigma \in \Sigma(r)$ and $i=1,2, \ldots, r$, we have

$$
\mathcal{L}_{V_{i}(S), \sigma(\sigma)}^{\left(L_{\alpha}, h\right)}=\sum_{j=1}^{r} b_{j}(\sigma, \alpha) s_{i}^{j}=\beta_{i}(S ; \sigma, \alpha),
$$

where $h$ is an arbitrary Hermitian metric on $L_{\alpha}$. Moreover we also have, for $\sigma \in \Sigma(r)$ and $i=1,2, \ldots, r$, 


$$
\mathcal{L}_{V_{i}(S), o(\sigma)}^{g^{\prime}}=\left(\begin{array}{cccc}
q_{i}^{1}(S ; \sigma) & & & 0 \\
& q_{i}^{2}(S ; \sigma) & & 0 \\
0 & & \ddots & \\
& & & q_{i}^{r}(S ; \sigma)
\end{array}\right)
$$

with respect to a basis $\left\{\left(\partial / \partial z^{1}(\sigma)\right)_{o(\sigma)}, \ldots,\left(\partial / \partial z^{r}(\sigma)\right)_{o(\sigma)}\right\}$ of $T_{o(\sigma)}^{1,0} X_{P}$, where $g^{\prime}$ is an arbitrary Hermitian metric on $X_{\Sigma}$. Together with (4.3) and (4.4), Fact 2.3 immediately implies the theorem.

As a corollary of this theorem, we obtain the following:

COROLlaRY 4.5. Let $X_{\Sigma}$ be an $r$-dimensional compact toric manifold associated with a complete non-singular fan $\Sigma, S \in G L(r, \boldsymbol{C})$ a non-degenerate non-singular matrix, $\alpha_{a}: \Sigma(1) \rightarrow \boldsymbol{Z}, a=1,2, \ldots, k$, and $b_{1}, b_{2}, \ldots, b_{k} \in N$ with $b_{1}+b_{2}+\cdots+b_{k}=r$. Then we have

$$
\begin{aligned}
& \left(c_{1}\left(L_{\alpha_{1}}\right)^{b_{1}} \cup c_{1}\left(L_{\alpha_{2}}\right)^{b_{2}} \cup \cdots \cup c_{1}\left(L_{\alpha_{k}}\right)^{b_{k}}\right)\left[X_{\Sigma}\right] \\
& =\sum_{\sigma \in \Sigma(r)} \frac{\prod_{a=1}^{k} \beta_{i}\left(S ; \sigma, \alpha_{a}\right)^{b_{a}}}{\prod_{j=1}^{r} q_{i}^{j}(S ; \sigma)}
\end{aligned}
$$

for any $i=1,2, \ldots, r$. In particular, for $\alpha: \Sigma(1) \rightarrow Z$ and $i=1,2, \ldots, r$, we have

$$
\mu_{c_{1}\left(L_{\alpha}\right)}=\frac{\sum_{\sigma \in \Sigma(r)} \frac{\beta_{i}(S ; \sigma, \alpha)^{r-1} \sum_{j=1}^{r} q_{i}^{j}(S ; \sigma)}{\prod_{j=1}^{r} q_{i}^{j}(S ; \sigma)}}{\sum_{\sigma \in \Sigma(r)} \frac{\beta_{i}(S ; \sigma, \alpha)^{r}}{\prod_{j=1}^{r} q_{i}^{j}(S ; \sigma)}}
$$


PROOF. Applying Theorem 4.2 to $\mathcal{C}_{L_{\alpha_{1}}^{\lambda_{1}} \otimes L_{\alpha_{2}}^{\lambda_{2}} \otimes \cdots \otimes L_{\alpha_{k}}^{\lambda_{k}}}^{c_{1}^{r}}\left(V_{i}(S)\right)$, we obtain

$$
\begin{aligned}
& \sum_{b_{1}+\cdots+b_{k}=r} \frac{r !}{b_{1} ! \cdots b_{k} !} \lambda_{1}^{b_{1}} \cdots \lambda_{k}^{b_{k}}\left(c_{1}\left(L_{\alpha_{1}}\right)^{b_{1}} \cup \cdots \cup c_{1}\left(L_{\alpha_{k}}\right)^{b_{k}}\right)\left[X_{\Sigma}\right] \\
& =\sum_{\sigma \in \Sigma(r)} \frac{\left(\sum_{a=1}^{k} \lambda_{a} \beta_{i}\left(S ; \sigma, \alpha_{a}\right)\right)^{r}}{\prod_{j=1}^{r} q_{i}^{j}(S ; \sigma)} .
\end{aligned}
$$

By comparing the coefficients of $\lambda_{1}^{b_{1}} \lambda_{2}^{b_{2}} \cdots \lambda_{k}^{b_{k}}$ in the equation above, we obtain the formula (4.5.1). The formula (4.5.2) is straightforward from the definition (1.2) of $\mu_{\eta}$ and the formula (4.5.1).

In view of Theorems 2.4 and 4.2 and Corollary 4.5 combined with the equalities

$$
\sum_{j=0}^{r}(-1)^{j}\left(\begin{array}{l}
r \\
j
\end{array}\right)(r-2 j)^{k}= \begin{cases}0 & \text { if } k=0,1, \ldots, r-1, r+1, \\
2^{r} r ! & \text { if } k=r,\end{cases}
$$

we can prove the following combinatorial formula for the Bando-Calabi-Futaki character of a compact toric manifold:

COROLlaRy 4.6. Let $X_{\Sigma}$ be an $r$-dimensional compact toric manifold associated with a complete non-singular fan $\Sigma$ and $S \in G L(r, \boldsymbol{C})$ a non-degenerate non-singular matrix. If $\alpha: \Sigma(1) \rightarrow \boldsymbol{Z}$ is ample, then, for $i=1,2, \ldots, r$, we have

$$
\begin{aligned}
& \sqrt{-1} F_{X_{\Sigma}}^{c_{1}\left(L_{\alpha}\right)}\left(V_{i}(S)\right)=\sum_{\sigma \in \Sigma(r)} \frac{\beta_{i}(S ; \sigma, \alpha)^{r} \sum_{j=1}^{r} q_{i}^{j}(S ; \sigma)}{\prod_{j=1}^{r} q_{i}^{j}(S ; \sigma)} \\
& \left.-\frac{\sum_{\sigma \in \Sigma(r)} \frac{\beta_{i}(S ; \sigma, \alpha)^{r-1} \sum_{j=1}^{r} q_{i}^{j}(S ; \sigma)}{\prod_{j=1}^{r} q_{i}^{j}(S ; \sigma)}}{\sum_{\sigma \in \Sigma(r)} \frac{\beta_{i}(S ; \sigma, \alpha)^{r}}{\prod_{j=1}^{r} q_{i}^{j}(S ; \sigma)}}\right\} \sum_{\sigma \in \Sigma(r)} \frac{\beta_{i}(S ; \sigma, \alpha)^{r+1}}{\prod_{j=1}^{r} q_{i}^{j}(S ; \sigma)} .
\end{aligned}
$$


REMARK 4.7. (i) Let $X_{\Sigma}, \alpha$ and $S$ be as in Corollary 4.6. Then we have

$$
\begin{aligned}
& \left(\begin{array}{llll}
F_{X_{\Sigma}}^{c_{1}\left(L_{\alpha}\right)}\left(\tau_{1}\right), & F_{X_{\Sigma}}^{c_{1}\left(L_{\alpha}\right)}\left(\tau_{2}\right), & \ldots, & F_{X_{\Sigma}}^{c_{1}\left(L_{\alpha}\right)}\left(\tau_{r}\right)
\end{array}\right) \\
& \quad=\left(\begin{array}{llll}
F_{X_{\Sigma}}^{c_{1}\left(L_{\alpha}\right)}\left(V_{1}(S)\right), & F_{X_{\Sigma}}^{c_{1}\left(L_{\alpha}\right)}\left(V_{2}(S)\right), & \ldots, & F_{X_{\Sigma}}^{c_{1}\left(L_{\alpha}\right)}\left(V_{r}(S)\right)
\end{array}\right) S^{-1} .
\end{aligned}
$$

Therefore, in view of Corollary 4.6, we can calculate $F_{X_{\Sigma}}^{c_{1}\left(L_{\alpha}\right)}\left(\tau_{i}\right)$ for all $i=1,2, \ldots, r$.

(ii) Let $X_{\Sigma}$ and $\alpha$ be as in Corollary 4.6. Then by means of Theorem 1.9, Corollary 4.6 and the identity in (i), we can obtain the entire information about the Bando-Calabi-Futaki character $F_{X_{\Sigma}}^{c_{1}\left(L_{\alpha}\right)}$ of $\left(X_{\Sigma}, c_{1}\left(L_{\alpha}\right)\right)$.

\section{REFERENCES}

[1] M. Audin, The topology of torus actions on symplectic manifolds, Progr. Math. 93, Birkhäuser Verlag, Basel, Boston, Berlin, 1991.

[2] S. BANDO, An obstruction for Chern class forms to be harmonic, preprint.

[ 3 ] R. BотT, A residue formula for holomorphic vector-fields, J. Differential Geom. 1 (1967), 311-330.

[ 4 ] E. CALABI, Extremal Kähler metrics II, Differential geometry and complex analysis, 95-114, SpringerVerlag, Berlin, Heidelberg, New York, 1985.

[ 5 ] D. A. Cox, The homogeneous coordinate ring of a toric variety, J. Algebraic Geom. 4 (1995), 17-50.

[ 6 ] A. FUנIKI, On automorphism groups of compact Kähler manifolds, Invent. Math. 44 (1978), 225-258.

[ 7 ] W. Fulton, Introduction to toric varieties, Ann. of Math. Stud. 131, Princeton Univ. Press, Princeton, New Jersey, 1993.

[ 8 ] A. FUTAKI, An obstruction to the existence of Kähler Einstein metrics, Invent. Math. 73 (1983), 437-443.

[9] A. FUTAKI, On compact Kähler manifolds of constant scalar curvature, Proc. Japan Acad. 59 (1983), 401402 .

[10] A. FUTAKI, Kähler-Einstein metrics and integral invariants, Lecture Notes in Math. 1314, Springer-Verlag, Berlin, Heidelberg, New York, 1987.

[11] A. FUTAKI AND S. MORITA, Invariant polynomials of the automorphism group of a compact complex manifolds, J. Differential Geom. 21 (1985), 135-142.

[12] S. KobAYASHI, Differential geometry of complex vector bundles, Publ. Math. Soc. Japan 14, Iwanami, Tokyo and Princeton Univ. Press, Princeton, 1987.

[13] T. MABUCHI, An algebraic character associated with the Poisson brackets, Recent topics in differential and analytic geometry, 339-358, Adv. Stud. in Pure Math. 18-I, Kinokuniya and Academic Press, Tokyo and Boston, 1990.

[14] Y. NAKAGAWA, Combinatorial formulae for Futaki characters and generalized Killing forms of toric Fano orbifolds, The Third Pacific Rim Geometry Conference, 223-260, Internat. Press, Cambridge, MA, 1998.

[15] Y. NAKAGAWA, Bando-Calabi-Futaki characters of Kähler orbifolds, Math. Ann. 314 (1999), 369-380.

[16] T. ODA, Convex bodies and algebraic geometry: An introduction to the theory of toric varieties, Ergeb. Math. Grenzgeb. (3) 15, Springer-Verlag, Berlin, Heidelberg, New York, 1988.

[17] G. TIAN, Kähler-Einstein metrics on algebraic manifolds, Proc. C.I.M.E. conference on Transcendental methods in algebraic geometry, 143-185, Lecture Notes in Math. 1646, Springer-Verlag, Berlin, Heidelberg, New York, 1996.

Mathematical InStitute

FACULTY OF SCIENCE

TÔHOKU UNIVERSITY

SENDAI 980-8578

JAPAN

E-mail address: nakagawa@math.tohoku.ac.jp 